\title{
A Versatile Compressible Fluid Experiment
}

\section{Dr. William M. Clark, Worcester Polytechnic Institute}

Dr. William Clark is an associate professor in the Chemical Engineering Department at Worcester Polytechnic Institute. He holds a B.S. from Clemson University and a Ph.D. from Rice University, both in Chemical Engineering. He has taught thermodynamics, separation processes, and unit operations laboratory for over 25 years. In addition to research efforts in teaching and learning, he has conducted disciplinary research in separation processes. 


\title{
A Versatile Compressible Fluid Experiment
}

\begin{abstract}
We have developed a versatile new laboratory apparatus that can be used for teaching a variety of chemical engineering fundamentals. The new equipment is used in our unit operations lab to address misconceptions and a lack of experience with compressible fluids by studying pressure drop during air flow through a pipe. We extended the range of experiments that can be done with the apparatus by including a Coriolis meter, an anemometer, an inline heater, a PID temperature controller, a vortex tube, and a Tesla turbine. This poster describes how the new equipment can be used in unit ops lab and in other courses for teaching aspects of fluid flow, material and energy balances, thermodynamics, and process control.
\end{abstract}

\section{Introduction}

To accommodate recent increases in enrollment, we had a need for a relatively inexpensive new experiment in our unit operations laboratory. We also had a need to include hands-on experience with process control in our curriculum and a desire to provide more hands-on experience in our sophomore material and energy balance and thermodynamics courses. We developed a versatile new air flow experiment to accomplish all these goals. Having undergraduate students help design, construct, and test the new equipment as their senior theses also provided an excellent educational experience for the students involved.

As shown in Figure 1, the heart of the apparatus is a 10 foot section of $1 / 4$ inch schedule 40 stainless steel, straight, horizontal pipe and a differential pressure gage for studying pressure drop at various flow conditions. Compressed air from our house air line (usually between 65 and $75 \mathrm{psig}$ ) is regulated at pressures up to $60 \mathrm{psig}$ as it enters the system. Flows up to $11 \mathrm{SCFM}$ are possible depending on the operating pressure and are established via a combination of pressure regulator and flow control valve settings. Also shown in Figure 1, are four flow meters, an inline heater, a PID temperature controller, a vortex tube, and a turbine that have been included to facilitate a variety of further studies. This equipment was relatively inexpensive as shown in the Appendix where the make, model, and cost of each of the main components is presented.

In this poster we discuss the design and implementation of the various experiments afforded by the apparatus, our experience with the gas pressure drop experiment in unit operations lab, and our plans to use the equipment in other courses. 


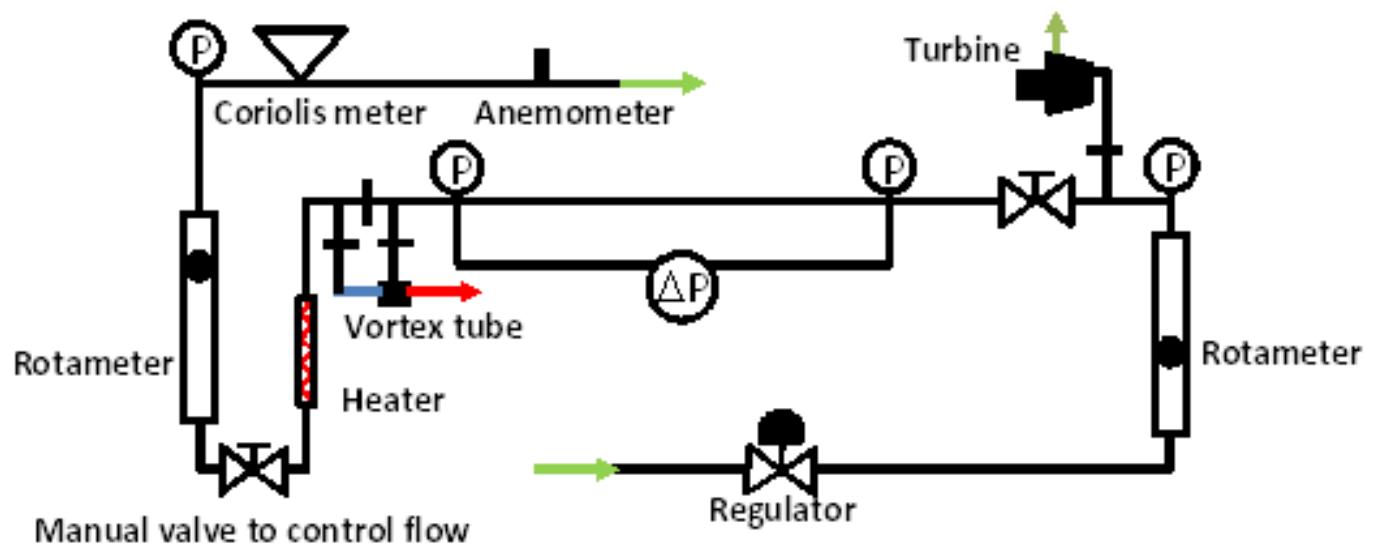

Figure1. Schematic diagram of air flow apparatus.

\section{Experiments and Example Results}

\section{Friction factor and pressure drop in a pipe}

In agreement with Luyben and Tuzla ${ }^{1}$, we had observed that our students lacked practical understanding of gas flows. We included a gas pressure drop experiment similar to the one they developed at Lehigh to expose students to the important observation that gas density affects pressure drop in pipe. To study compressible flow, students in our unit ops lab are instructed to determine the operating pressure and flow rate dependence of the pressure drop in the pipe. Most students recognize that varying the operating pressure at a fixed mass flow rate and then repeating this procedure for several different mass flow rates is a good way to proceed. Since adjustments in the flow control valve also affect the operating pressure and the operating pressure affects the flow meter readings, it usually takes some time and thought to learn how to accomplish this, however. The students are, therefore, advised to study the various flow measurement devices as described in the next section before attempting the pressure drop studies.

Once they learn how to measure and control flows and operating pressures, they can easily collect pressure drop data over a wide range of operating conditions using a Dwyer Instruments (model 4202), 0 to 2 psi, differential pressure gage. This allows them to generate a plot like Figure 2 showing that pressure drop increases with increasing flow at a fixed pressure but decreases with increasing pressure at a fixed flow rate. 


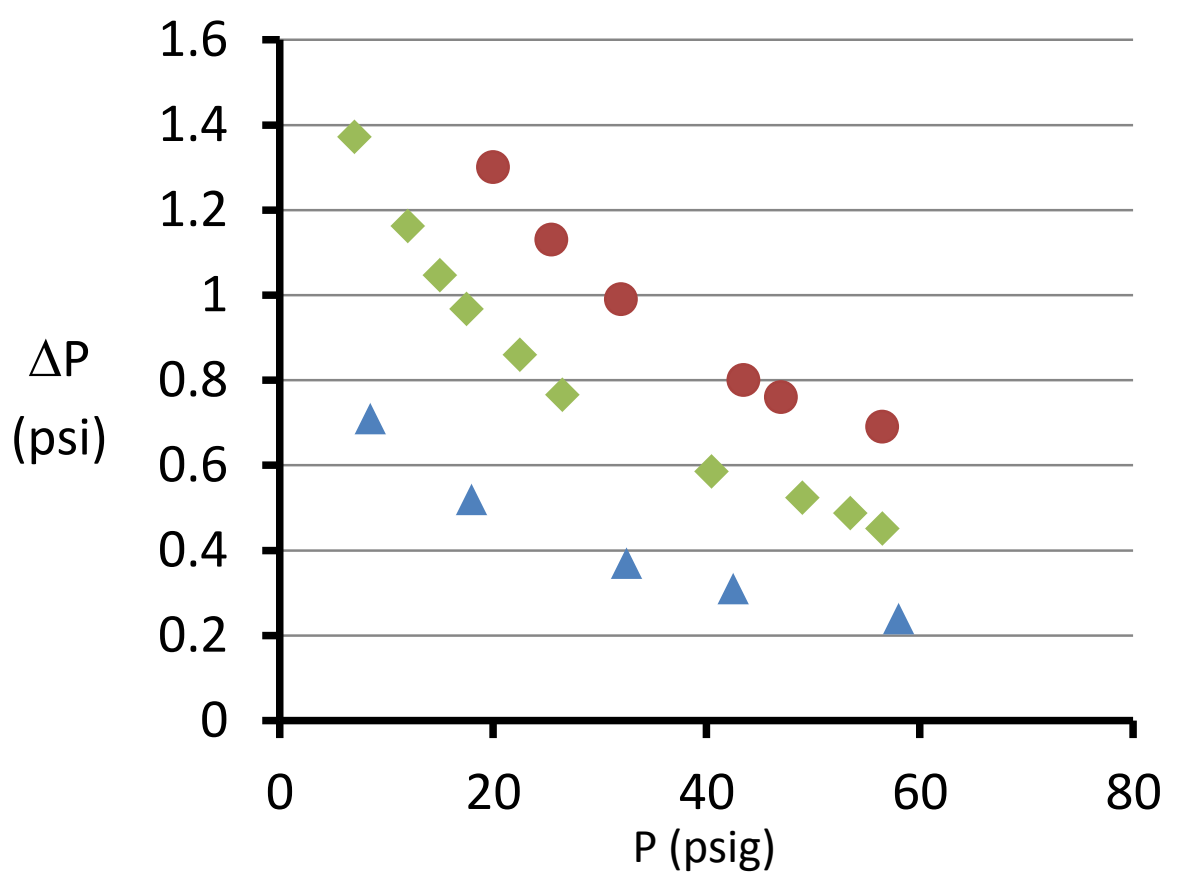

Figure 2. Pressure drop as a function of operating pressure for three mass flow rates: circles $=9$ SCFM, diamonds = 7 SCFM, triangles = 5 SCFM.

The data range covered in reference 1 was smaller than that shown here and there was some discussion as to whether the curves obtained should be linear or not. The reason for the curvature seen in Figure 2 can be clarified as follows. For a fixed mass flow rate and cross sectional area, the product of density and velocity must be constant. Therefore, both the Reynolds number and the Fanning friction factor

$$
f=\frac{\Delta P D}{2 L \rho V^{2}}
$$

are nearly constant. Equation 1, can be rearranged to

$$
\Delta P=K_{1} V=\frac{K_{2}}{\rho}
$$

where $\mathrm{K}_{\mathrm{i}}$ represents a constant. Using the relationship between density and pressure given by the ideal gas law, Equation 2 becomes

$$
\Delta P=\frac{K_{3}}{P}
$$

A linear relationship exists between pressure drop and 1/P (rather than $\mathrm{P}$ ). The lines with intersections at the origin shown in Figure 3 were obtained using 1/P in psia. 


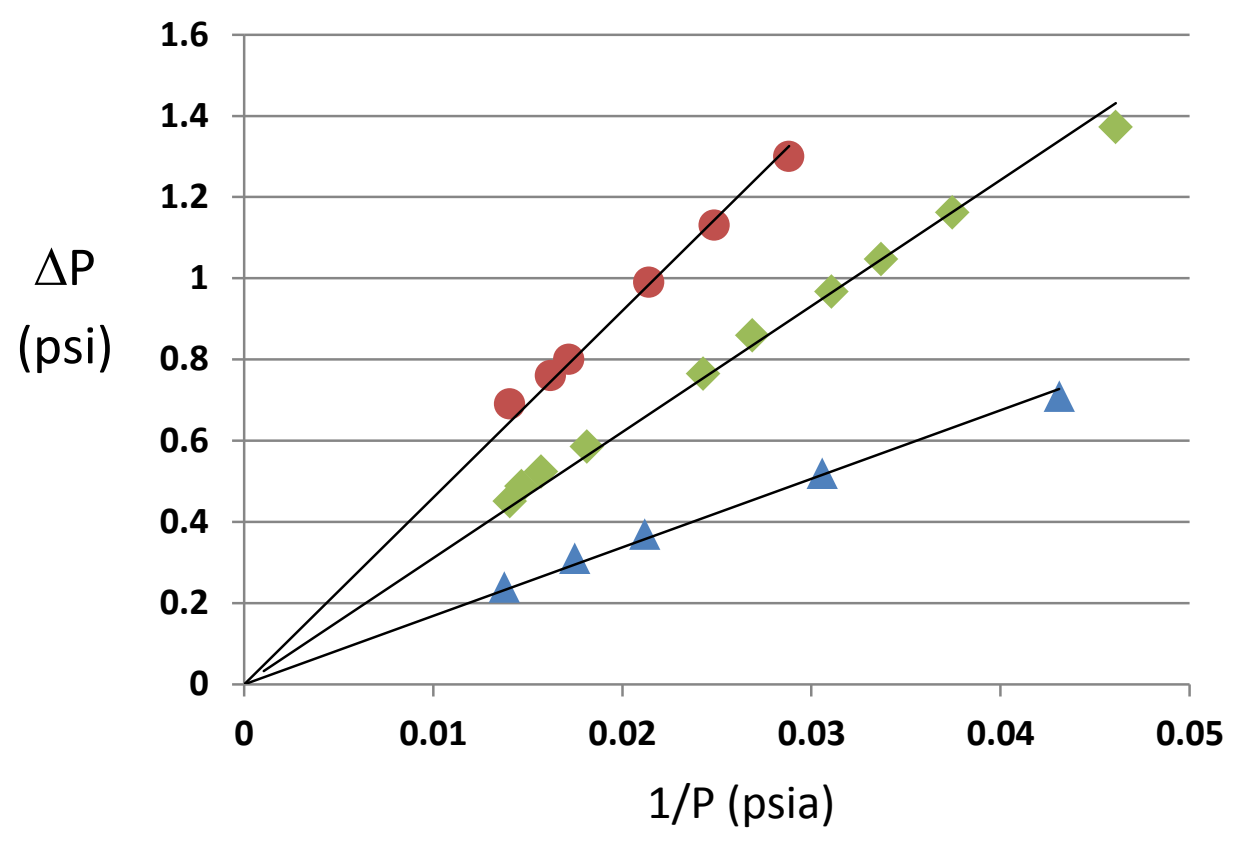

Figure 3. Pressure drop as a function of reciprocal pressure at three mass flow rates: circles $=9$ SCFM, diamonds = 7 SCFM, triangles = 5 SCFM.

It is of interest to note that, in two years of experience with this experiment in our lab course, only one student group out of 15 groups generated a plot like Figure 3 . This is perhaps understandable because students were explicitly asked to generate a plot of pressure drop versus operating pressure. It is somewhat disappointing, however, since they were also asked to analyze and discuss their results to the best of their ability.

Students are also encouraged to plot their pressure drop results on a Moody chart as shown in Figure 4. This solidifies the point that Reynolds number and friction factor are nearly constant at fixed mass flow rate. It also allows estimation of pipe roughness. Although more expensive than other materials that could be used, stainless steel is a good choice since it has a welldocumented, non-zero expected roughness that students can compare with their measured value. 


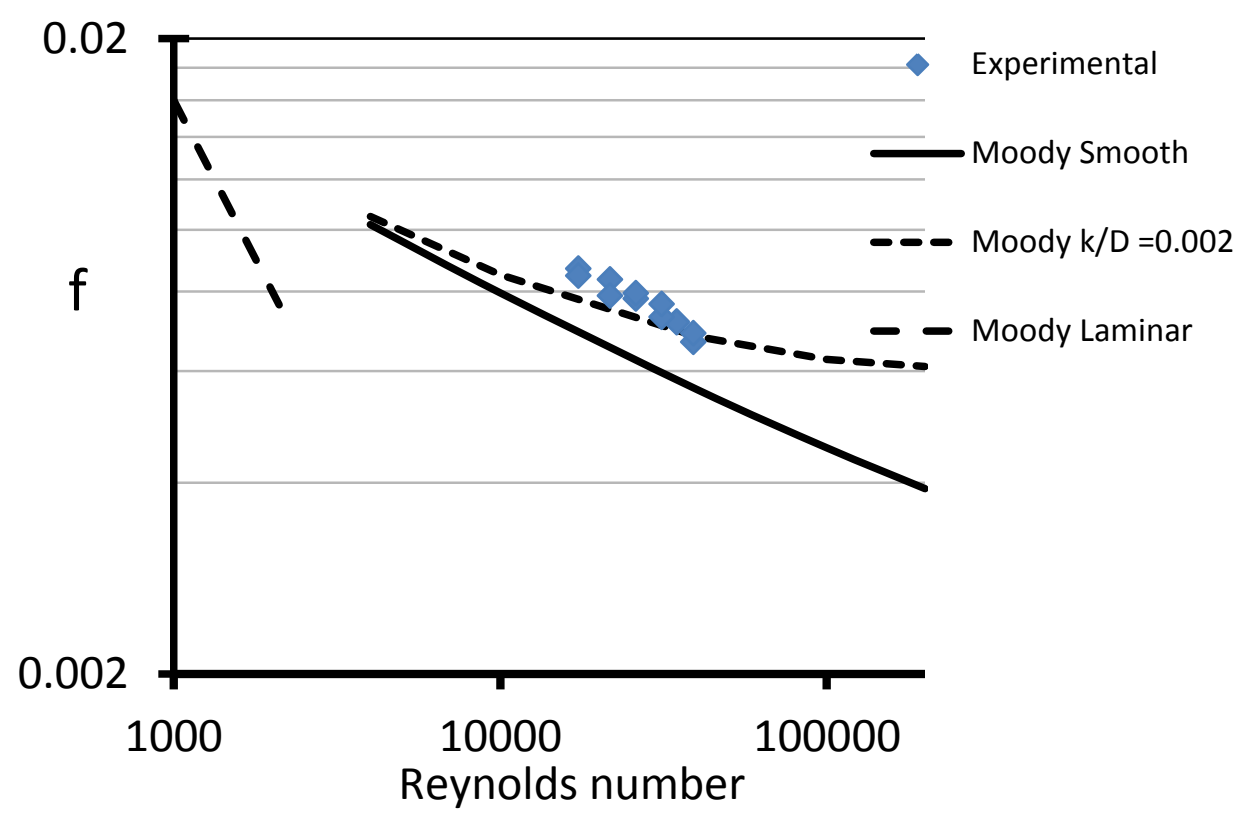

Figure 4. Moody chart showing measured and expected results for friction factor as a function of Reynolds number.

Written lab reports indicate that most students understand that higher flow rates and lower pressures result in higher pressure drops. Questions during oral reports have revealed, however, that not all students fully understand that the reason the pressure drop increases with decreasing pressure is that the concomitant density decrease results in an increasing velocity and it is the fluid velocity that governs the pressure drop. We have developed Comsol Multiphysics computer models of the air flow process to address this point and are pursuing it further as explained elsewhere ${ }^{2}$.

\section{Flow measurement}

Also similar to Luyben and Tuzla ${ }^{1}$, we utilize a rotameter near the system entrance to measure the air flow and teach students how to correct gas flow meter readings for conditions different from those used in calibration. In addition, we included a second rotameter after the flow control valve, near the system exit, to drive home the concept that the apparent flow rate depends on the operating conditions. Students have to convince themselves that the mass flow remains constant even though the two rotameters have vastly different readings. We have also included a Coriolis mass flow meter and a vane anemometer that give other readings that can be corrected to the same mass flow rate as shown in Table 1. The rotameters are Omega Engineering model FL1503A precision rotameters. The anemometer (model HHF141A-USB) was also obtained from Omega. The Coriolis meter is a Micro Motion model CMF025 that was generously donated to us by Micro Motion and Pond Technical Sales, Inc. 
Most students found that the Coriolis meter was the easiest to use to establish constant mass flow for their pressure drop measurements. Most students also understood that the higher pressure at the first rotameter resulted in a higher density and a lower velocity and therefore a lower apparent flow rate than at the second rotameter. It is not yet clear if students gained a good understanding of why the pressure correction utilizes the square root of the pressure ratio, however. We have developed a Comsol Multiphysics model that calculates the drag force on a rotameter float and plan to use it in the future to help clarify exactly how a variable area flow meter works ${ }^{2}$.

Table 1. Data and results for flow measurement by four different flow meters at steady state.

\begin{tabular}{|c|c|c|c|c|c|c|c|c|}
\hline Coriolis & \multicolumn{2}{|c|}{ Anemometer } & \multicolumn{3}{c|}{ Rotameter 1 } & \multicolumn{3}{c|}{ Rotameter 2 } \\
\hline Reading & Reading & Result & Reading & Pressure & Result & Reading & Pressure & Result \\
\hline SCFM & FPM & SCFM & CFM & PSIG & SCFM & CFM & PSIG & SCFM \\
\hline 10.8 & 3500 & 10.8 & 5.23 & 46 & 10.6 & 9.04 & 5 & 10.5 \\
\hline
\end{tabular}

\section{Heater and PID controller}

We installed a $400 \mathrm{~W}$ inline heater (Omega model AHP-5051) before the second rotameter to demonstrating the temperature dependence of our flow meter readings. To avoid overheating our system, we included a temperature controller (Omega model CN9000A) and controlled the maximum temperature to be $70{ }^{\circ} \mathrm{C}$. This allows for a modest but measurable density (and velocity) change at the downstream flow meters. We also installed thermocouples at various locations in the system and a watt meter to measure the energy input to the heater. Although we have asked our unit operations students to verify the simple energy balance this affords, we plan to ask sophomore thermodynamics students to use this equipment for that purpose in the future. Future plans also include using the heater and temperature controller for teaching practical aspects of process control in a separate unit operations lab experiment or in our control class. The Omega PID controller can be used for this purpose, but we found it difficult to use when varying the process control parameters. We developed a LabView PID controller with easily adjustable parameters that can be used as an alternative to the Omega controller. Figure 5 shows the LabView control panel that displays our temperature measurements along with the measurements from our Coriolis meter and anemometer. 


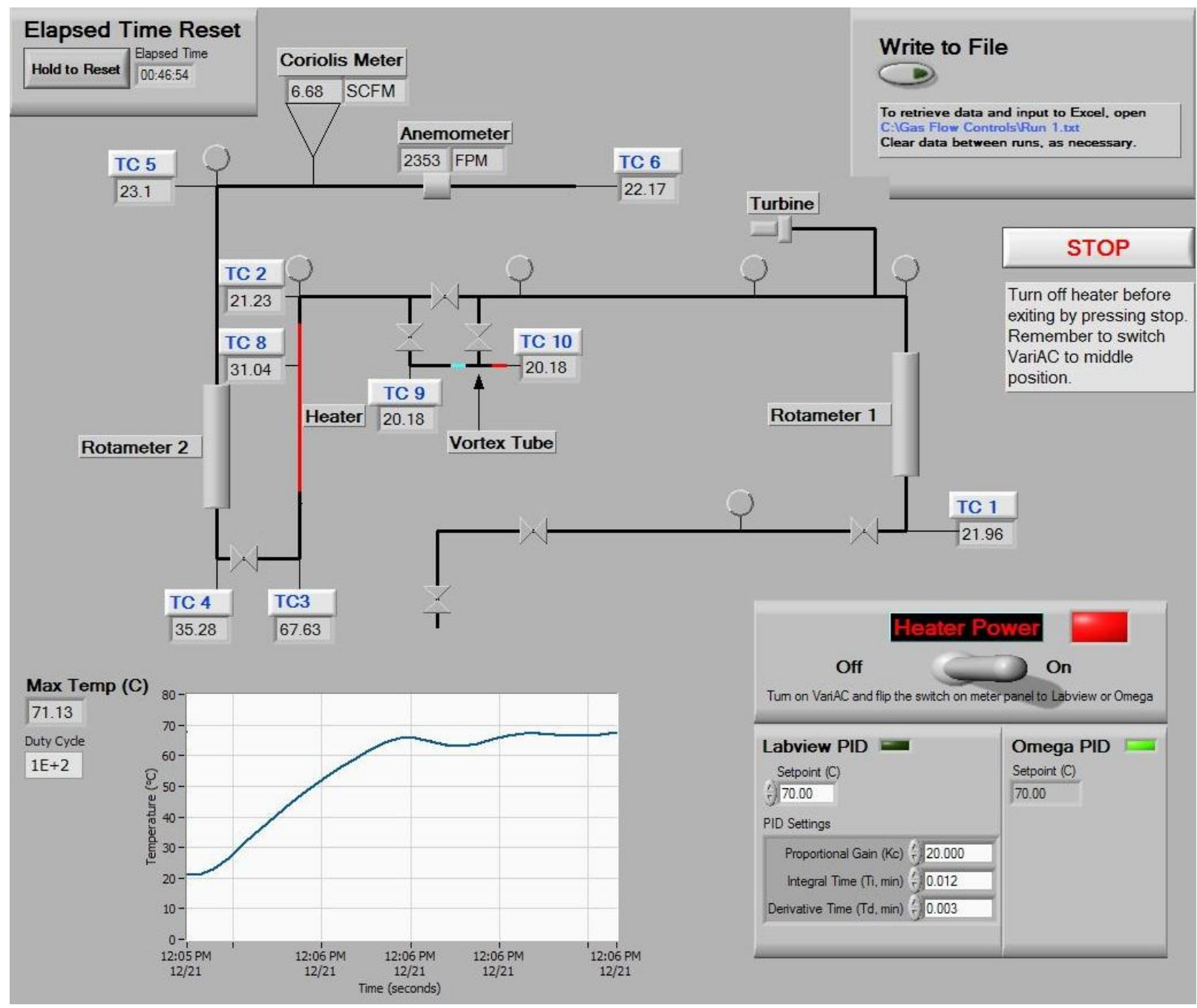

Figure 5. LabView control panel for heater and temperature control experiments.

\section{Vortex tube}

With no moving parts or applied fields, a vortex tube takes an inlet air stream at room temperature and separates it into a cold stream and a warm stream. Adjusting a valve on the warm stream varies the flow rate and temperature of the two outlet streams. We obtained a vortex tube from AiRTX International ${ }^{3}$ and installed it in our system such that the cold product stream flows past the heater and the downstream flow meters while the warm product stream exits to the room as shown in Figures 1, 5, and 6. This not only provides a wider range of operating conditions for the heater and temperature control experiments but also allows for an interesting experimental study of the $1^{\text {st }}$ and $2^{\text {nd }}$ laws of thermodynamics for a flow system.

Table 2 shows example data and results that demonstrate that energy is conserved and the $2^{\text {nd }}$ law is not violated in this novel device. $\Delta \mathrm{H}$ and $\Delta \mathrm{S}$ values were evaluated assuming ideal gas with temperature dependent heat capacity. 


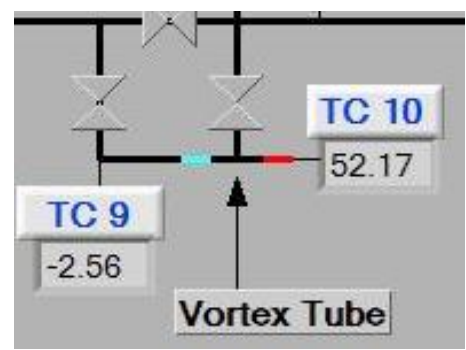

Figure 6. Example temperature readings with vortex tube in operation.

Table 2. Example vortex tube data and results.

\begin{tabular}{|c|c|c|c|c|c|}
\hline & Inlet & Cold stream & Warm stream & Total \\
\hline Air flow & $\mathrm{mol} / \mathrm{s}$ & $\begin{array}{c}0.142 \\
\text { measured }\end{array}$ & $\begin{array}{c}0.079 \\
\text { measured }\end{array}$ & $\begin{array}{c}0.063 \\
\text { by mass balance }\end{array}$ & 0.142 \\
\hline $\mathrm{T}$ & ${ }^{\circ} \mathrm{C}$ & 21.86 & -2.56 & 52.17 & \\
\hline $\mathrm{P}$ & $\mathrm{psia}$ & 71.7 & 24.7 & 14.7 & 0.23 \\
\hline$\Delta \mathrm{H}$ & $\mathrm{J}$ & & -56.25 & 56.48 & 1.515 \\
\hline$\Delta \mathrm{S}$ & $\mathrm{J} / \mathrm{K}$ & & 0.503 & 1.012 & \\
\hline
\end{tabular}

Although we have not required our unit operations lab students to study the vortex tube, those who have ventured to play with it found it very interesting. We plan to have sophomore thermodynamics students use this equipment in the future and want to determine if seeing (and feeling) the vortex tube in operation will have a measurable effect on learning and satisfaction for classical thermodynamics students.

\section{Tesla turbine}

Another interesting device that we believe will be an aid to learning classical thermodynamics is a small air-powered turbine that we obtained from Gyroscope.com ${ }^{4}$. The Tesla turbine takes input air at known temperature, pressure and flow rate and incorporates a three phase motor to generate electricity to light three 10 (or 20) W light bulbs. Figure 7 shows a measurement of RPMs of the device. As shown in Figure 8, we have installed a voltmeter, an ammeter, and a frequency meter (calibrated to measure RPM) to monitor the electrical output from the turbine. Accurate electric meters with digital readouts and the capability to connect to a computer were purchased from Laurel Electronics. These included a model L10002MRV3 voltmeter, a model L10006MRA4 ammeter, and a model L50002-FR frequency meter. 
We believe that seeing the turbine in operation and taking measurements to determine its efficiency will aid in the understanding of concepts like shaft work, isentropic work, and lost work. Students can observe the turbine shaft turning the generator and can see more power being produced as RPMs increase with increasing air flow. Example data and results for measuring the efficiency are given in Table 3.

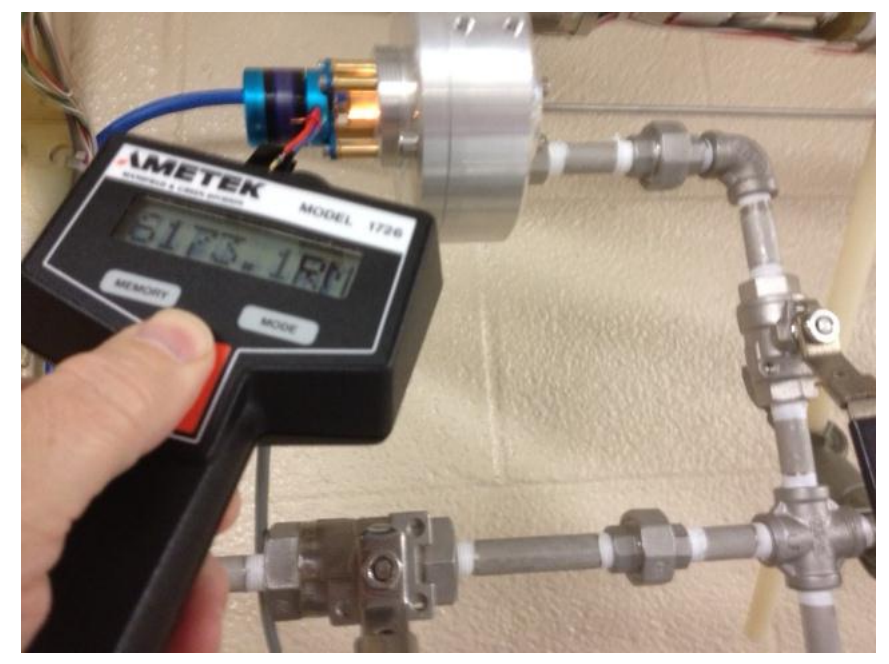

Figure 7. RPM measurement on our Tesla turbine.

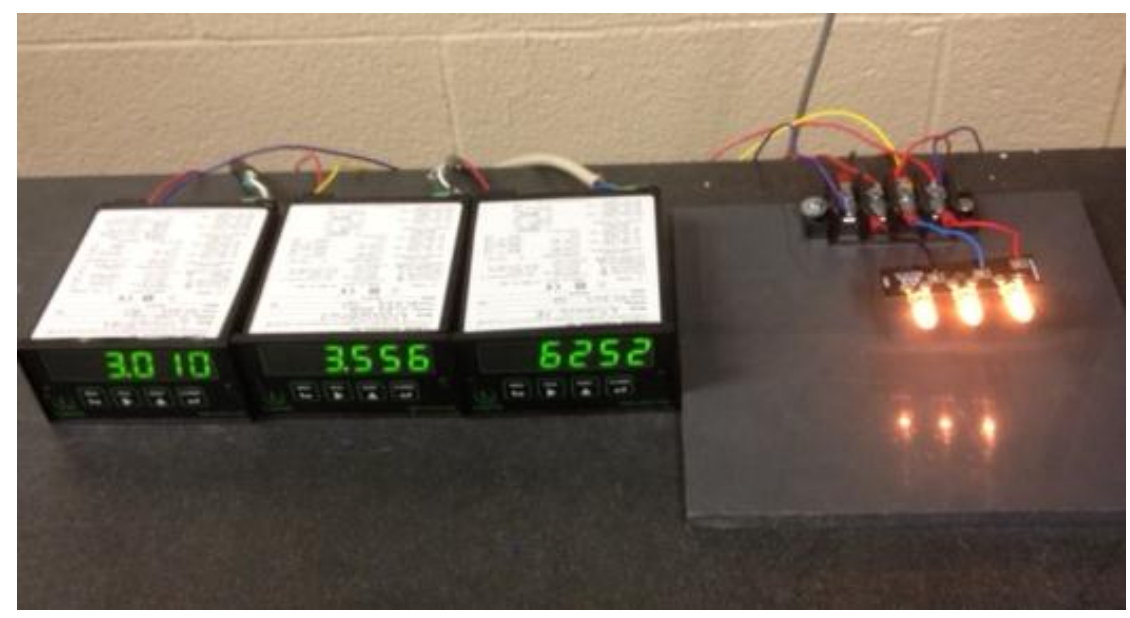

Figure 8. Electrical signal measurements (A, V, RPM) and light output from our turbine. 
Table 3. Data and results for turbine efficiency measurement.

\begin{tabular}{|l|l|l|l|l|l|}
\hline Voltage & $\mathrm{V}$ & 6.6 & $\mathrm{RPM}$ & & 10457 \\
\hline Current & $\mathrm{A}$ & 2.14 & Electrical work, We & $\mathrm{W}$ & 24.46 \\
\hline $\mathrm{P}_{\text {in }}$ & psia & 37.7 & Shaft work, Ws & $\mathrm{W}$ & 24.61 \\
\hline $\mathrm{P}_{\text {out }}$ & psia & 4.7 & $\Delta \mathrm{S}$ & $\mathrm{W} / \mathrm{K}$ & 1.465 \\
\hline $\mathrm{T}_{\text {in }}$ & ${ }^{\circ} \mathrm{C}$ & 22.54 & $\mathrm{~T}_{\text {isentropic }}$ & $\mathrm{K}$ & 225.6 \\
\hline $\mathrm{T}_{\text {out }}$ & ${ }^{\circ} \mathrm{C}$ & 18.27 & $\mathrm{~W}_{\text {isentropic }}$ & $\mathrm{W}$ & 401.4 \\
\hline Flow & $\mathrm{SCFM}$ & 10.05 & Efficiency, $\eta$ & $\%$ & 6.1 \\
\hline Flow & mol/s & 0.198 & $\mathrm{~W}_{\text {lost }}$ & $\mathrm{W}$ & 430 \\
\hline
\end{tabular}

Due to the way we measured volts and amps from the three phase generator, the electrical work was calculated as

$$
W_{e}=V A \sqrt{3}
$$

The reported shaft work is simply the actual enthalpy change for the air given by

$$
W_{s}=n \Delta H=n \int_{T_{\text {in }}}^{T_{\text {out }}} C_{p} d T
$$

The shaft work was found to be similar to the electrical work produced for this run. The turbine efficiency was determined as

$$
\eta=\frac{W_{e}}{W_{\text {isentropic }}} \cdot 100 \%
$$

where $\mathrm{W}_{\text {isentropic }}$ is the work that would be done by an adiabatic and reversible (constant $\mathrm{S}$ ) expansion of air at the given flow rate and inlet and outlet pressures. $\mathrm{T}_{\text {isentropic }}$ is the outlet temperature that would be reached if such a process took place. The isentropic work was determined by evaluating Equation 5 between $\mathrm{T}_{\text {in }}$ and $\mathrm{T}_{\text {isentropic. The lost work takes into account }}$ work that could have been obtained via heat transfer with the surroundings at $T_{\mathrm{o}}=294 \mathrm{~K}$.

$$
W_{\text {lost }}=T_{o} \Delta S
$$

The results in Table 3 were found assuming ideal gas behavior. A more complete analysis including residual properties for non-ideal gas behavior made essentially no difference as would be expected at the low operating pressure. Increasing the operating pressure increased the air flow rate through the turbine and that increased both the electrical work produced and the 
isentropic work. The turbine efficiency also increased with increasing flow rate but our system was limited to conditions that yielded less than $10 \%$ efficiency. We considered installing a dedicated high-capacity air compressor to try to reach higher efficiencies, but we estimated that the maximum efficiency that can be reached before the turbine exceeds the recommended RPM maximum of 20,000 is about $20 \%$. We concluded that having students make measurements at the available conditions and then make further calculations at assumed higher pressure and flow values is preferable to incurring the expense to install a larger air compressor.

\section{Conclusion}

A multifaceted air flow apparatus can be built relatively inexpensively and used for teaching a variety of chemical engineering fundamentals including aspects of fluid flow, material and energy balances, classical thermodynamics, and process control. Based on written and oral reports from students who used the equipment in unit operations lab, we believe that it helps students learn to appreciate the need to correct for temperature and pressure of flow readings for compressible fluids and get a physical feel for how these factors change density, velocity, and volumetric flow rate in the pipe. Students also seem to appreciate that pressure drop varies with both flow rate and operating pressure for compressible fluids, but some fail to recognize the mechanism for the effect of operating pressure. We are looking forward to using the equipment for teaching thermodynamic analysis of a vortex tube and a Tesla turbine to sophomores and evaluating how hands-on experience with these devices affects their satisfaction and learning.

\section{Acknowledgements}

The outstanding work of the following undergraduate students who contributed to the design, implementation, and testing of the new apparatus is gratefully acknowledged: Mary Hesler, Ryan Vautrin, Ross Yaylaian, Taylor Mazzali, Jared Brown, Michael Lynch, Rodrigo Ma, Ben Baranowski, Thaddeus Adams, and Michael Hoyt. Successful construction of the equipment would not have been possible without the help and expertise of machinist Giacomo Ferraro and electronics engineer Douglas White. We also thank Joe Pilsbury, Pond Technical Sales, and Micro Motion, Inc. for their generous donation of a Coriolis mass flow meter.

\section{References}

1. William L. Luyben and Kemal Tuzla, Gas Pressure-Drop Experiment, Chem. Eng. Education, 44 (3), pp. 183188, Summer (2010).

2. William M. Clark, Comsol Models Combined with Experiments to Teach Compressible Fluid Concepts, Proceedings 2011 Comsol Conference Boston, October 2011. 
3. www.airtx.com

4. www.gyroscope.com

Appendix. Make, model, and cost of various components of the versatile air flow apparatus.

The table below shows the approximate cost of the various components of the apparatus. The cost of pipe and fittings and labor to assemble the equipment has not been included. Although we recommend stainless steel pipe because it has a non-negligible roughness, less expensive pipe can be used. Thermocouples and the computer interface for reading them have also not been itemized. Assuming that a continuous supply of $65+$ psig air is available, it can be seen that the basic air flow apparatus, made up of the items in the first four rows along with the associated pipes and fitting, is relatively inexpensive. Choices can be made regarding addition of the other components depending on the budget and needs of the user.

\begin{tabular}{|c|c|c|c|}
\hline Component & Vendor & Model & Cost \\
\hline Pressure regulator & Omega Engineering & PRG501-60 & $\$ 240$ \\
\hline Rotameter (x2) & Omega Engineering & FL-1503A-B & $\$ 650(\mathrm{x} 2)$ \\
\hline Pressure gage $(\mathrm{x} 4)$ & Dwyer Instruments & SGI-F0424N & $\$ 75 \quad(x 4)$ \\
\hline Differential pressure gage & Dwyer Instruments & 4202 (0-2 psi) & $\$ 366$ \\
\hline Anemometer & Omega Engineering & HHF143A-USB & $\$ 1040$ \\
\hline Coriolis meter & Micro Motion & CMF025 & $\begin{array}{l}\$ 9738 \text { (donated to us by } \\
\text { Pond Technical Sales) }\end{array}$ \\
\hline Heater & Omega Engineering & AHP-5051 & $\$ 80$ \\
\hline Temperature controller & Omega Engineering & CN9120A & $\$ 210$ \\
\hline $\begin{array}{l}\text { Multimeter (for heater } \\
\text { watts measurement) }\end{array}$ & Murata Power Solutions & ACM20-2-AC1-R-C & $\$ 65$ \\
\hline Vortex tube & AiRTX International & 20010 & $\$ 164$ \\
\hline Turbine & Gyroscope.com & $\begin{array}{l}\text { Tesla turbine-closed loop } \\
\text { hybrid }\end{array}$ & $\$ 353$ \\
\hline Ammeter & Laurel Electronics & L10006RMA4,CBL05 & $\$ 385$ \\
\hline Volt meter & Laurel Electronics & L10002RMV3 & $\$ 350$ \\
\hline Frequency meter & Laurel Electronics & L50002FR & $\$ 320$ \\
\hline
\end{tabular}

